\title{
Laparoscopic Approach for Pancreatic Leiomyosarcoma with Metachronous Liver Metastasis
}

\author{
Woohyung Lee, M.D.', Jae Yool Jang, M.D.', Soon-Chan Hong, M.D., Ph.D.', Chi-Young Jeong, M.D., Ph.D.' \\ Department of Surgery, 'Gyeongsang National University Changwon Hospital, Changwon, ${ }^{2} G y e o n g s a n g$ National University Hospital, Gyeongsang National \\ University Postgraduate School of Medicine, Jinju, Korea
}

\begin{abstract}
Although pancreatic leiomyosarcoma (PLM) is a rare malignant pancreatic cancer, it usually shows aggressive biological features such as invasion to an adjacent organ or distant metastasis at the time of diagnosis. Radical resection is the best treatment modality but effective chemotherapies have not been identified. A 58-year-old female was referred to us complaining of intermittent left upper quadrant abdominal discomfort. Imaging studies revealed a $10-\mathrm{cm}$ mass in the pancreatic tail. The patient underwent laparoscopic distal pancreatectomy with splenectomy, and the pathological findings were consistent with PLM. Imaging studies 14 months after surgery revealed multiple liver metastases. Because the patient was young with a sufficient remnant liver, we performed laparoscopic metastatectomy without any postoperative complications. Patients with PLM need frequent checkups, even after curative resection. The role of laparoscopic resection should be confirmed in the future.
\end{abstract}

Keywords: Pancreatic cancer, Leiomyosarcoma, Laparoscopy, Pancreatectomy, Liver resection
Received October 19, 2016

Revised December 1, 2016

Accepted February 8, 2017

Corresponding author

Chi-Young Jeong

Department of Surgery, Gyeongsang

National University Hospital,

Gyeongsang National University

Postgraduate School of Medicine, 79

Gangnam-ro, Jinju 52727, Korea

Tel: $+82-55-750-8096$

Fax: +82-55-750-9244

E-mail: drjcy@hanmail.net

Copyright @ 2017 The Journal of Minimally Invasive Surgery. All rights reserved.
This is an Open Access article distributed under the terms of the Creative Commons Attribution Non-Commercial License (http:// creativecommons.org/licenses/by-nc/4.0/) which permits unrestricted non-commercial use, distribution, and reproduction in any medium, provided the original work is properly cited.

\section{INTRODUCTION}

Pancreatic leiomyosarcoma (PLM) accounts for only $0.1 \%$ of malignant pancreatic cancers. It is associated with aggressive features, such as invasion of an adjacent organ or distant metastasis at the time of diagnosis, in about one-half of patients with PLM. ${ }^{1}$ Radical resection is still the best treatment option for localised PLM, and patients who underwent curative resection showed better survival than patients who received palliative care. ${ }^{2}$ However, one-quarter of patients had metastasis after curative resection and the management of patients with metastatic lesions has not been ascertained. Effective systemic chemotherapies have not been identified. Recent progress in laparoscopic techniques and equipment has enabled surgeons to perform advanced laparoscopic surgery. Furthermore, previous studies have shown better postoperative results and similar oncological outcomes after laparoscopic pancreatectomy for malignant pancreatic tumours, compared with conventional open surgery. ${ }^{3,4}$ However, the laparoscopic approach for PLM has not yet been reported. This article presents a case of PLM with metachronous liver metastasis that was treated laparoscopically.

\section{CASE REPORT}

A 59-year woman visited a primary clinic after experiencing abdominal discomfort for several months. Abdominal ultrasonography showed a pancreatic mass and she was referred to our hospital for further evaluation. The patient had undergone right thyroidectomy for nodular goiter. There were 
no specific features on physical examination, and laboratory tests were within the normal ranges, including tumour markers. Abdominal computed tomography (CT) revealed a 10$\mathrm{cm}$ mass in the pancreatic tail, abutting the stomach, spleen, left adrenal gland, and left kidney. The magnetic resonance imaging (MRI) findings suggested a solid pseudopapillary neoplasm or neuroendocrine tumour (Fig. 1). However, the patient had no facial flushing or diarrhea, which are typical symptoms associated with neuroendocrine tumors. Laparoscopic distal pancreatectomy was performed, and the operative findings showed no evidence of a mass originating from any other adjacent organ. Operation time and estimated blood loss (EBL) were $255 \mathrm{~min}$ and $300 \mathrm{ml}$, respectively. The pathological findings were of a $10-\mathrm{cm}$ pancreatic tumor with a free surgical margin. Microscopically, the tumor consisted of uniform-sized spindle cells with abundant eosinophilic cytoplasm, and showed highly cellularity and nuclear atypia. Frequent mitotic activity was seen with up to 22 mitoses per 10 high-power fields (HPFs). There were areas of degenerative and focal necrosis within the lesion. Immunohistochemical analysis showed that the tumour cells were positive for cluster of differentiation (CD) 34, smooth muscle actin, and vimentin, and negative for S-100, cytokeratin, and CD117 (Fig. 2). The tumor was consistent with PLM with high-grade malignancy. The abdominal drain was removed after checking the abdominal CT on postoperative day 7. The patient was followed up without adjuvant chemoradiation therapy after surgery. Abdominal MRI and positron emission tomography revealed multiple hepatic metastases 14 months after surgery (Fig. 3). Because chemoradiation therapy is ineffective for PLM, we recommended surgical treatment of the liver metastases. Preoperative liver function tests, including the indocyanine green retention rate at $15 \mathrm{~min}$, were within the normal range. The patient underwent laparoscopic right hemihepatectomy with segment 1, 4 tumorectomy (Fig. 4). Operation time and EBL were $485 \mathrm{~min}$ and $400 \mathrm{ml}$, respectively. She was discharged on postoperative day 17 without hepatic failure or other postoperative complications. No recurrence was found at her latest visit to the outpatient department at 5 months after surgery.

\section{DISCUSSION}

Primary mesenchymal tumors of the pancreas account for $1 \% \sim 2 \%$ of pancreatic tumors. They can be classified based on their histological origins as schwannoma (22.2\%), leiomyosarcoma (18.6\%), myofibroblastic tumour (15.4\%), and hamartoma $(8.1 \%)^{5,6}$ PLMs originate from the smooth muscle of the pancreatic vessel wall or ductal wall ${ }^{7}$ and are extremely rare, accounting for $0.1 \%$ of pancreatic tumors. Differentiating invasive leiomyosarcoma from tumors of the stomach, duodenum, and retroperitoneum is crucial in diagnosing primary PLM.

It was previously reported that PLM occurs in similar numbers of males and females (male-to-female ratio: 1.06) and the median age at diagnosis is 53 (14 87) years. The most common symptoms are abdominal mass $(29 / 63,46.0 \%)$, pain $(25 / 63$, $36.6 \%)$, and weight loss $(19 / 63,30.1 \%)$. In terms of tumor site, the incidence was similar in the pancreatic head and bodytail. $^{8}$

Although PLMs cannot be differentiated from other pancreatic masses based on imaging findings alone, some features should prompt the clinician to suspect PLM. For example, a mosaic echo pattern is observed on ultrasonography, a large heterogeneous mass with necrotic, calcified regions and greater vascular enhancement is visible on CT scans, while on MRI the mass is isointense to muscle on T1-weighted images and hyperintense on T2-weighted images. ${ }^{9}$ Although fine needle biopsy guided by endoscopic ultrasonography is helpful for diagnosing PLM, some clinicians are concerned about the risk of bleeding or tract seeding of malignant cells.

Histologically, PLM is characterised by interlaced, pleomorphic spindle cells. However, this finding is insufficient for the diagnosis of PLM. Accurate diagnosis requires immuno-
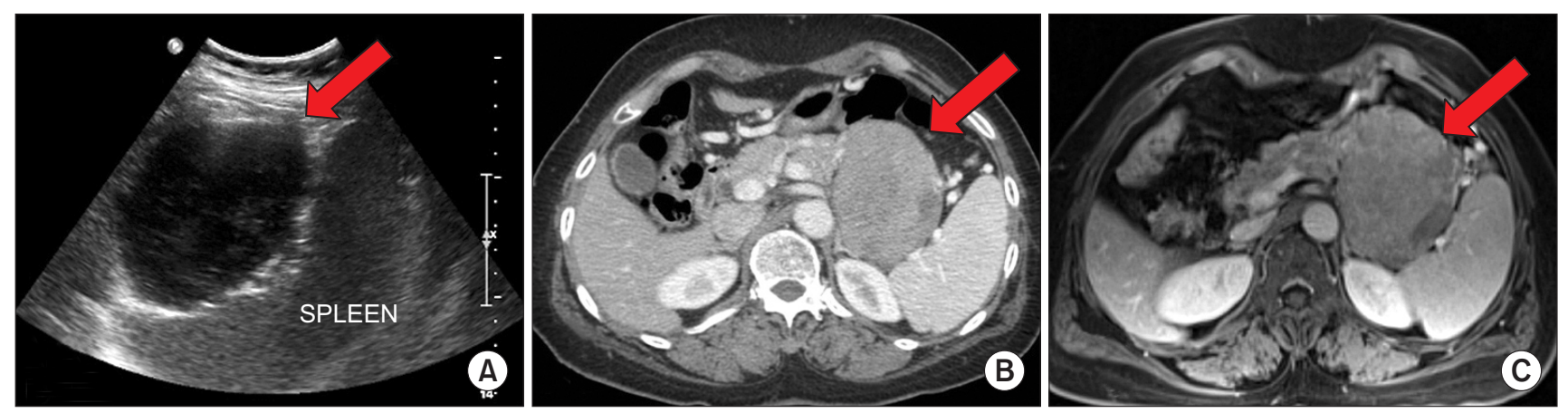

Fig. 1. Imaging studies show a large heterogeneous mass arising from the body and tail of the pancreas (arrow). (A) Ultrasonography, (B) computed tomography, and (C) magnetic resonance imaging. 

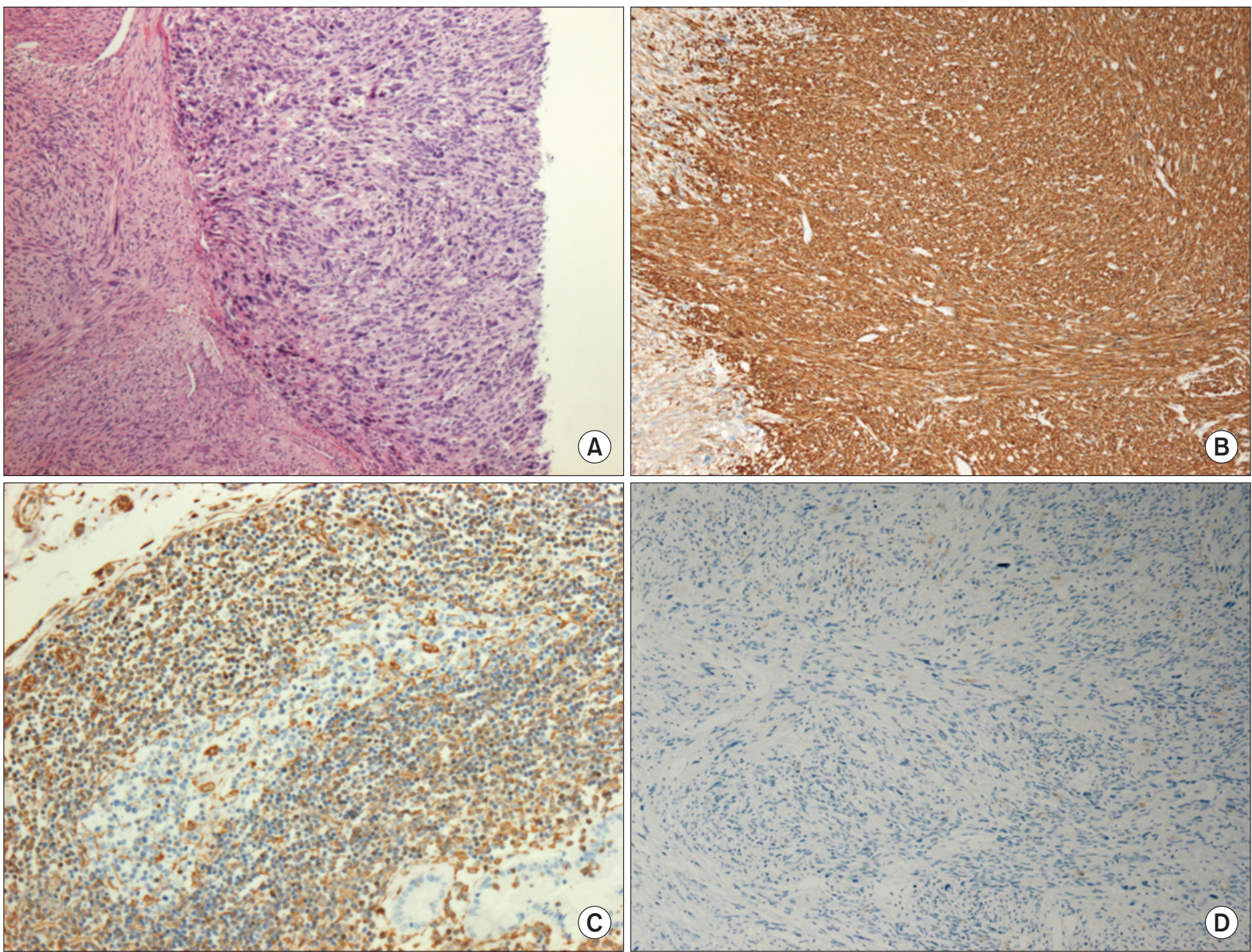

Fig. 2. Microscopic and immunohistochemical features of pancreatic leiomyosarcoma. (A) Spindle cells show increased mitotic activity with haematoxylin and eosin staining. $(B \sim D)$ The tumour cells are positive for smooth muscle actin $(B)$ and vimentin $(C)$, and negative for $S-100(D)$.
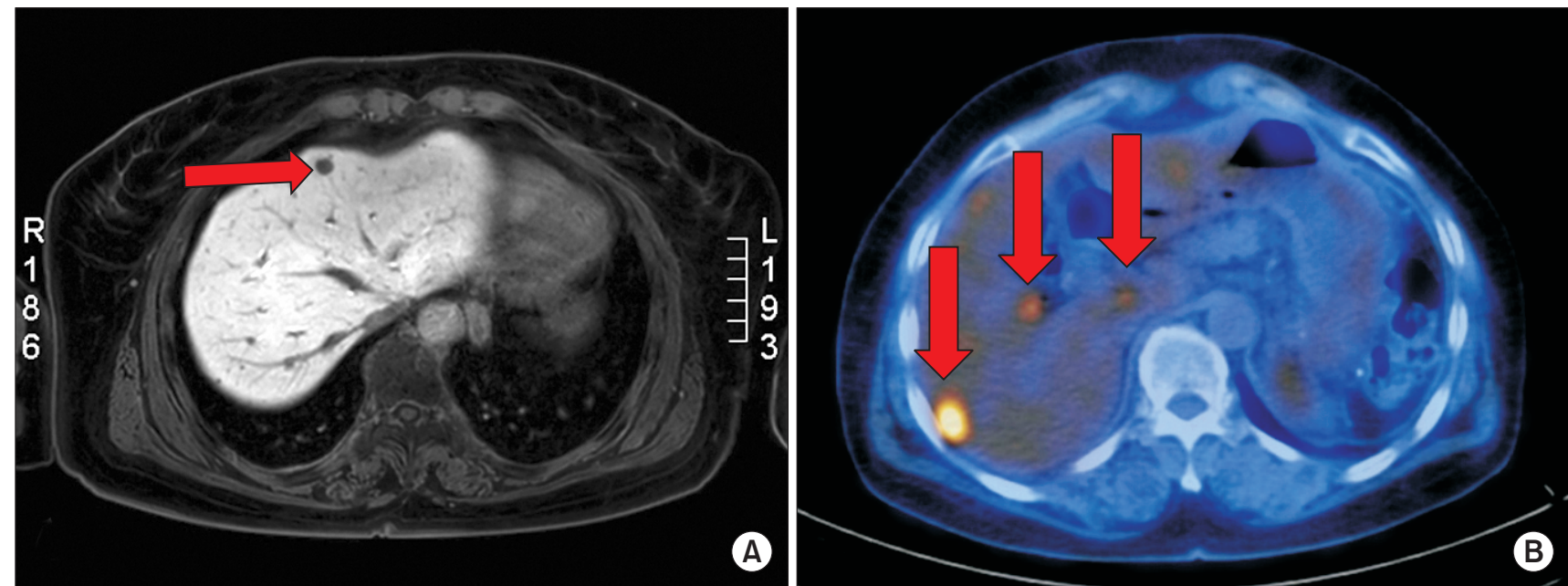

Fig. 3. Follow-up imaging studies reveal multiple liver metastases (arrows). (A) Magnetic resonance imaging and (B) positron emission tomography. 

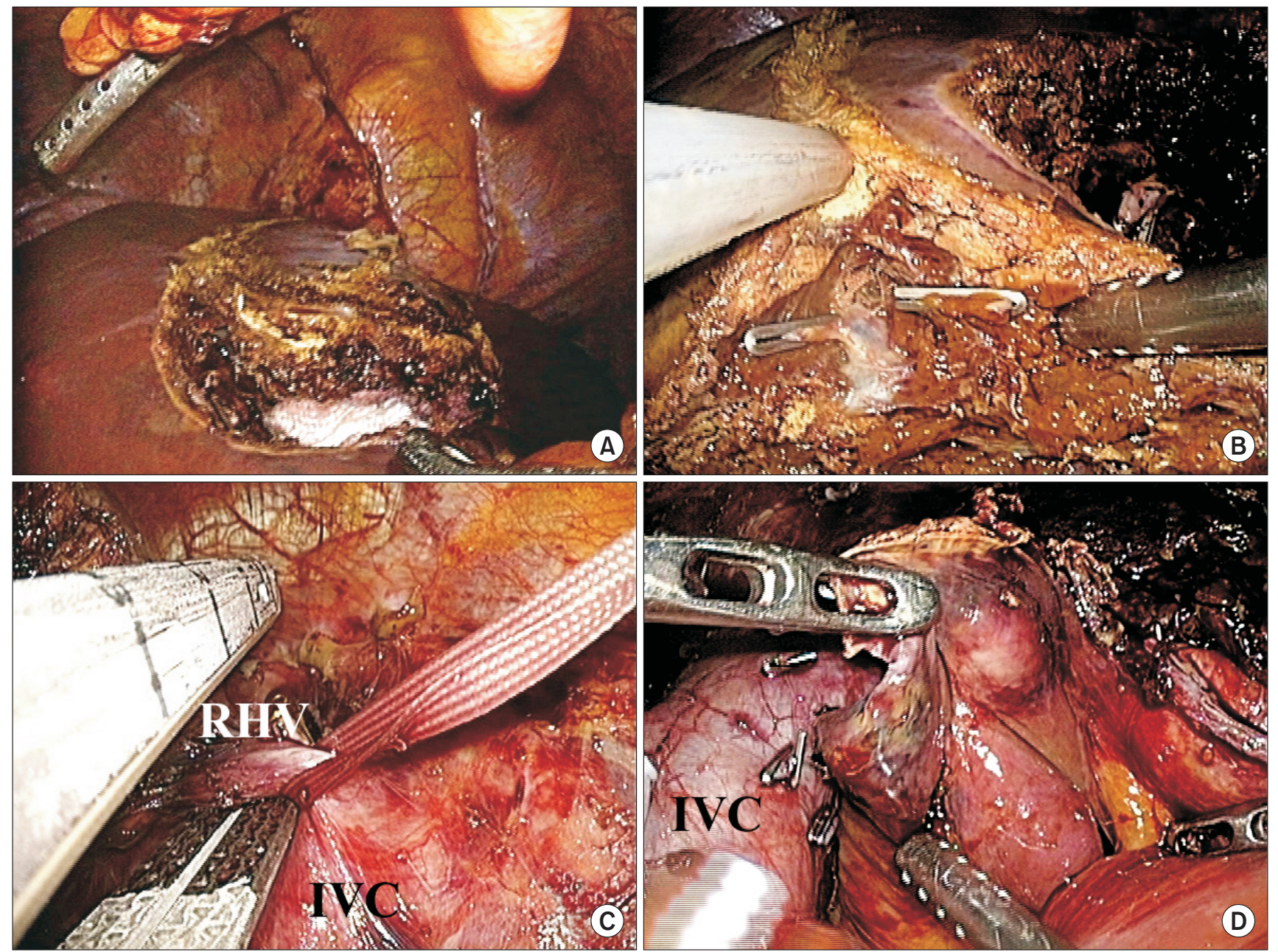

Fig. 4. Laparoscopic right hemihepatectomy with segment 1, 4 tumorectomy. (A) Tumor located in segment 4 was dissected. (B) Liver parenchymal dissection along the Cantile's line. (C) Right hepatic vein was separated by stapler. (D) Tumor was located in caudate lobe.

histochemical staining. The diagnostic criteria for leiomyosarcoma are positive staining for muscle markers such as smooth muscle actin, vimentin, or desmin; and negative staining for epithelial and neural markers such as cytokeratin, epithelial membrane antigen, $\mathrm{S}-100$ protein, or CD117. ${ }^{10}$

PLM displays aggressive biological features. It was reported that $25 \%$ of patients had distant metastasis and $20 \%$ of patients had invasion to an adjacent organ at the time of diagnosis. ${ }^{8}$ However, lymph node metastasis is less common in patients with PLM than in patients with other cancers. ${ }^{10}$ The median survival time was reported to be 48 months and adverse prognostic factors for survival were non-radical resection, invasion of adjacent organs, or a mitotic count of more than $10 \mathrm{HPFs}^{8}{ }^{8}$

Although patients undergo curative resection, 25\% of patients develop metastases in the liver, lung, or peritoneum. There are five reported cases of PLM with metachronous liver metastasis, including the present report. All patients experi- enced liver metastasis within 6 44 months after pancreatectomy and none of the patients underwent liver metastatectomy. Our patient underwent laparoscopic right hemihepatectomy with segment 1, 4 tumorectomy to treat liver metastasis after laparoscopic distal pancreatectomy for PLM. Metastatectomy is not widely accepted as a general treatment for recurrent PLM. However, effective chemotherapies after pancreatic resection for PLM have not been identified. Therefore, surgical treatment may be considered for highly selected patients, especially young patients who are in good general health and have localized recurrence. However, it is necessary to evaluate the oncologic results of this type of treatment in large-scale studies.

Recent improvements in laparoscopic surgery have enabled surgeons to perform advanced laparoscopic procedures. The role of laparoscopic surgery for pancreatic malignant tumours has also expanded. Several studies have reported better post- 
operative results and similar oncological outcomes for malignant lesions after laparoscopic pancreatectomy, compared with conventional open surgery. In addition, the indications for laparoscopic liver resection have expanded to malignant lesions, including liver metastasis from colorectal cancer, and major laparoscopic liver resection is increasingly being performed at experienced centers. ${ }^{11}$ Although we have described successful laparoscopic treatment of a patient with PLM and liver metastasis, further studies are needed to confirm the role of laparoscopic treatment in this setting.

\section{REFERENCES}

1) Moletta L, Sperti C, Beltrame V, et al. Leiomyosarcoma of the Pancreas with Liver Metastases as a Paradigm of Multimodality Treatment: Case Report and Review of the Literature. J Gastrointest Cancer 2012;43 Suppl 1:S246-250.

2) Hur YH, Kim HH, Park EK, et al. Primary leiomyosarcoma of the pancreas. J Korean Surg Soc 2011;81 Suppl 1:S69-73.

3) Shin SH, Kim SC, Song KB, et al. A comparative study of laparoscopic vs. open distal pancreatectomy for left-sided ductal adenocarcinoma: a propensity score-matched analysis. J Am Coll Surg 2015;220:177-185.

4) de Rooij T, Jilesen AP, Boerma D, et al. A nationwide comparison of laparoscopic and open distal pancreatectomy for benign and malignant disease. J Am Coll Surg 2015;220:263-270.e261.

5) Vanderpuye V, Clegg-Lamptey JN, Yarney J, Aryeetey NA. Metastatic Primary Leiomyosarcoma of the Pancreas to the Liver: Report of a Surgically Treated Case. J Gastrointest Cancer 2012;43 Suppl 1:S70-72.

6) Kim JY, Song JS, Park H, et al. Primary mesenchymal tumors of the pancreas: single-center experience over 16 years. Pancreas 2014:43:959-968.

7) Feinberg SB, Margulis AR, Lober P. Roentgen findings in leiomyosarcoma of the pancreas. Minn Med 1957;40:505-506.

8) Xu J, Zhang T, Wang T, You L, Zhao Y. Clinical characteristics and prognosis of primary leiomyosarcoma of the pancreas: a systematic review. World J Surg Oncol 2013;11:290.

9) Kocakoc E, Havan N, Bilgin M, Atay M. Primary pancreatic leiomyosarcoma. Iran J Radiol 2014;11:e4880.

10) Zhang H, Jensen MH, Farnell MB, Smyrk TC, Zhang L. Primary leiomyosarcoma of the pancreas: study of 9 cases and review of literature. Am J Surg Pathol 2010;34:1849-1856.

11) Wakabayashi G, Cherqui D, Geller DA, et al. Recommendations for laparoscopic liver resection: a report from the second international consensus conference held in Morioka. Ann Surg 2015;261:619-629. 